\title{
Effect of preoperative statins in patients without coronary artery disease who undergo cardiac surgery
}

\author{
Minoru Tabata, MD, MPH, Zain Khalpey, MD, PhD, Lawrence H. Cohn, MD, Fredrick Y. Chen, MD, PhD, \\ R. Morton Bolman, III, MD, and James D. Rawn, MD
}

\begin{abstract}
Objective: 3-hydroxy-3-methylglutaryl coenzyme A reductase inhibitors (statins) have been shown to have pleiotropic effects in addition to their lipid-lowering properties. Some studies have shown the beneficial effect of preoperative statins on operative outcomes in coronary artery bypass grafting. However, the effect of preoperative statins in patients without coronary artery disease who undergo cardiac surgery remains poorly defined.
\end{abstract}

\begin{abstract}
Methods: We performed a retrospective review of 1389 consecutive patients undergoing cardiac valve surgery between January of 2002 and December of 2005. Patients undergoing concomitant coronary artery bypass surgery and those with a history of myocardial infarction and coronary interventions were excluded. Of this cohort, 363 patients were receiving a statin preoperatively and 1026 patients were not. Propensity scores were constructed with patients' demographics, clinical data, and the year of procedure. Generalized estimating equations, including the propensity score as a covariate, were used to investigate whether preoperative statin use is associated with improved operative outcomes.

Results: The crude operative mortality rate was $0.8 \%$ and $2.3 \%$, the incidence of stroke was $1.7 \%$ and $2.9 \%$, and the incidence of perioperative myocardial infarction was $2.2 \%$ and $2.4 \%$ in the statin and non-statin groups, respectively. Generalized estimating equations showed that preoperative statin use is associated with lower mortality (odds ratio: $0.25,95 \%$ confidential interval: $0.12-0.54$ ). Preoperative statin use was not significantly associated with an incidence of stroke (odds ratio: $0.48,95 \%$ confidential interval: $0.19-1.22$ ) or perioperative myocardial infarction (odds ratio: $0.91,95 \%$ confidential interval: $0.43-1.91$ ) in this cohort.
\end{abstract}

Conclusion: Preoperative use of statins may improve operative outcomes in patients without coronary artery disease who undergo cardiac surgery.

3-hydroxy-3-methylglutaryl coenzyme A reductase inhibitors (statins) are potent cholesterol-lowering medications used primarily for hypercholesterolemia. They have been shown to have cholesterol-independent pleiotropic effects: to improve endothelial function, stabilize atherosclerotic plaques, decrease oxidative stress and inflammation, and inhibit the thrombogenic response. ${ }^{1}$

Statins have been shown to reduce the incidence of coronary events and stroke ${ }^{2}$ and are effective in the prevention of major cardiac events in coronary artery disease $(\mathrm{CAD}) .^{3-9} \mathrm{In}$ the field of cardiac surgery, some retrospective studies have shown that preoperative statin use reduces mortality and morbidity after coronary artery bypass grafting (CABG). ${ }^{10-12}$ However, the effect of preoperative statins in non-CABG cardiac surgery is unclear. Pleiotropic effects of statins could attenuate the adverse effect of surgical invasiveness and the proinflammatory milieu that ensues after

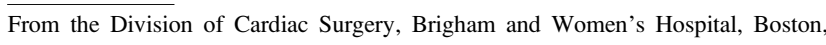
Mass.

James Rawn reports equity ownership in McKesson.

Received for publication Dec 16, 2007; revisions received Feb 26, 2008; accepted for publication March 7, 2008.

Address for reprints: Minoru Tabata, MD, MPH, Division of Cardiothoracic Surgery, Columbia University, College of Physicians and Surgeons, 177 Fort Washington

Avenue, MHB 7-435, New York, NY 10032 (E-mail: mtabata@post.harvard.edu).

J Thorac Cardiovasc Surg 2008;136:1510-3

$0022-5223 / \$ 34.00$

Copyright (c) 2008 by The American Association for Thoracic Surgery

doi:10.1016/j.jtcvs.2008.03.061 cardiopulmonary bypass $(\mathrm{CPB})$ regardless of the presence of CAD.

The aim of this study is to assess the association of preoperative statins with early operative outcomes of cardiac surgery without CAD. We hypothesize that preoperative use of statins reduces operative mortality, stroke, and perioperative myocardial infarction (PMI) in patients without CAD who undergo cardiac surgery.

\section{MATERIALS AND METHODS}

\section{Study Design and Data Collection}

We conducted a retrospective cohort study reviewing 1389 patients who underwent cardiac valve surgery between January of 2002 and December of 2005. We included the most recent 4 years to minimize time-related confounding factors.

All adult patients undergoing any cardiac valve surgery with CPB at Brigham and Women's Hospital during this period were included in this study. We exclusively extracted clinical data on the patients undergoing cardiac valve surgery within our database. Exclusion criteria included age less than 18 years old, emergency salvage surgery, significant CAD on preoperative angiography, previous myocardial infarction, previous $\mathrm{CABG}$, previous or planned percutaneous coronary interventions, concomitant CABG, and concomitant ventricular assist device implantation. We evaluated coronary arteries with preoperative angiography for all patients except those who were younger than 40 years of age and who had no apparent risk factors for atherosclerotic disease.

Preoperative use of statins was defined as being prescribed any class of statins at the time of admission. By this definition, we found 363 patients taking statins before surgery and 1026 patients not taking statins. A total of 197 patients $(54.3 \%)$ were taking atorvastatin, 126 patients $(34.7 \%)$ 
were taking simvastatin, 21 patients $(5.8 \%)$ were taking pravastatin, 8 patients $(2.2 \%)$ were taking fluvastatin, 7 patients $(1.9 \%)$ were taking lovastatin, and 4 patients $(1.1 \%)$ were taking rosuvastatin (Table 1$)$. Routine preoperative management included continuing preoperatively prescribed statins until the day before surgery; no statins were newly prescribed before surgery, and statins were restarted after surgery in all patients who were taking statins before surgery.

Operative mortality was defined as a death within 30 days after surgery or during the same hospitalization. Stroke was defined as a central neurologic deficit persisting postoperatively for more than 72 hours during the hospitalization. PMI was defined as postoperative elevation of creatinine kinase-MB isoenzyme of more than $100 \mathrm{U} / \mathrm{L}$ (20 times higher than the upper normal limit in our laboratory) at any time during the immediate postoperative period during the current hospitalization. Creatinine kinase-MB isoenzyme was routinely assessed in all cases. No patients had postoperative coronary angiography or intervention in this cohort.

Aortic valve disease, mitral valve disease, and tricuspid valve disease were considered present if surgical replacement or repair of each valve was performed. Other cardiac disease was considered present if the operation included concomitant non-valve surgery, such as congenital heart surgery, atrial fibrillation surgery, and thoracic aortic surgery. All other variables were defined on the basis of the definitions provided by the Society of Thoracic Surgery National Adult Cardiac Surgery Database version 2.52 .

All data were collected from the Brigham and Women's Hospital Cardiac Surgery Database and medical records. This study was approved by the institutional review board (IRB Protocol 2004p000028) of Brigham and Women's Hospital.

\section{Data Analysis}

Patients' demographic, clinical characteristics, and the year of procedure were compared between the statin and non-statin groups. We used an unpaired $t$ test for continuous variables and a chi-square or Fisher's exact test for categoric variables. Continuous variables are expressed as mean \pm standard deviation.

We used propensity scores for risk adjustment because the numbers of outcome events were low and there were multiple confounding factors. ${ }^{13}$ We built a propensity score model for taking preoperative statins, including all variables listed in Table 2 to adjust for those confounding factors. The C-index of the propensity score model was 0.77 . Furthermore, to assess whether preoperative statin use is associated with the incidence of operative mortality, stroke, and PMI, we used the propensity scores in regression adjustment. ${ }^{14}$ We used generalized estimating equations to adjust for surgeon cluster effects. ${ }^{15}$ We included a propensity score and the CPB time

TABLE 1. Preoperative statins and doses

\begin{tabular}{lclc}
\hline Statin and dose & No. of patients & Statin and dose & No. of patients \\
\hline Atorvastatin & 197 & Pravastatin & 21 \\
$5 \mathrm{mg}$ & 4 & $10 \mathrm{mg}$ & 2 \\
$10 \mathrm{mg}$ & 103 & $20 \mathrm{mg}$ & 10 \\
$20 \mathrm{mg}$ & 62 & $40 \mathrm{mg}$ & 8 \\
$30 \mathrm{mg}$ & 1 & $80 \mathrm{mg}$ & 1 \\
$40 \mathrm{mg}$ & 20 & Fluvastatin & 8 \\
$60 \mathrm{mg}$ & 1 & $20 \mathrm{mg}$ & 2 \\
$80 \mathrm{mg}$ & 6 & $40 \mathrm{mg}$ & 6 \\
Simvastatin & 126 & Lovastatin & 7 \\
$10 \mathrm{mg}$ & 16 & $20 \mathrm{mg}$ & 6 \\
$20 \mathrm{mg}$ & 65 & $80 \mathrm{mg}$ & 1 \\
$40 \mathrm{mg}$ & 35 & Rosuvastatin & 4 \\
$80 \mathrm{mg}$ & 10 & $20 \mathrm{mg}$ & 4 \\
\hline
\end{tabular}

TABLE 2. Preoperative characteristics of all patients

\begin{tabular}{|c|c|c|c|}
\hline Characteristics & $\begin{array}{c}\text { Statin } \\
(n=363)\end{array}$ & $\begin{array}{l}\text { Non-statin } \\
(\mathrm{n}=1026)\end{array}$ & $P$ value \\
\hline Age & $66.6 \pm 11.8$ & $59.6 \pm 15.5$ & $<.001$ \\
\hline Male & $184(50.7 \%)$ & $573(55.8 \%)$ & .090 \\
\hline Body mass index & $28.1 \pm 6.0$ & $26.6 \pm 5.7$ & $<.001$ \\
\hline History of smoking & $184(50.7 \%)$ & $431(42.0 \%)$ & .004 \\
\hline Current smoking & $26(7.2 \%)$ & $95(9.3 \%)$ & .224 \\
\hline $\begin{array}{c}\text { Family history } \\
\text { of CAD }\end{array}$ & $76(20.9 \%)$ & $170(16.6 \%)$ & .061 \\
\hline Diabetes & $56(15.4 \%)$ & $273(35.8 \%)$ & $<.001$ \\
\hline Renal insufficiency & $21(5.8 \%)$ & $45(4.4 \%)$ & .282 \\
\hline Hemodialysis & $3(0.8 \%)$ & $4(0.4 \%)$ & .313 \\
\hline Hypertension & $226(62.3 \%)$ & $418(40.7 \%)$ & $<.001$ \\
\hline COPD & $63(6.9 \%)$ & $114(11.1 \%)$ & .002 \\
\hline Peripheral vascular disease & $53(14.6 \%)$ & $137(13.4 \%)$ & .552 \\
\hline History of CVA & $25(6.9 \%)$ & $36(3.5 \%)$ & .007 \\
\hline Recent CVA & $0(0 \%)$ & $1(0.1 \%)$ & .552 \\
\hline Active endocarditis & $5(1.4 \%)$ & $31(3.0 \%)$ & .090 \\
\hline NYHA class 3 or 4 & $185(17.8 \%)$ & $297(28.9 \%)$ & .078 \\
\hline History of CHF & $122(33.6 \%)$ & $334(32.6 \%)$ & .713 \\
\hline Previous cardiac surgery & $40(11.0 \%)$ & $142(13.8 \%)$ & .171 \\
\hline Atrial fibrillation & $22(6.1 \%)$ & $48(4.7 \%)$ & .301 \\
\hline Ejection fraction & $57.1 \pm 11.9$ & $57.3 \pm 11.0$ & .743 \\
\hline Aortic valve disease & $231(63.6 \%)$ & $516(50.3 \%)$ & $<.001$ \\
\hline Mitral valve disease & $154(42.4 \%)$ & $576(56.1 \%)$ & $<.001$ \\
\hline Tricuspid valve disease & $38(10.5 \%)$ & $117(11.4 \%)$ & .466 \\
\hline Other cardiac disease & $102(28.1 \%)$ & $303(29.5 \%)$ & .606 \\
\hline Non-elective surgery & $72(19.8 \%)$ & $167(16.3 \%)$ & .123 \\
\hline Preoperative IABP & $0(0 \%)$ & $2(0.2 \%)$ & .400 \\
\hline Immunosuppressive therapy & $17(4.7 \%)$ & $46(4.5 \%)$ & .875 \\
\hline Aspirin & $192(52.9 \%)$ & $250(24.4 \%)$ & $<.001$ \\
\hline Beta blockers & $129(35.5 \%)$ & $182(17.7 \%)$ & $<.001$ \\
\hline ACE inhibitors & $59(16.3 \%)$ & $107(10.4 \%)$ & .003 \\
\hline Performed in 2003 & $85(23.4 \%)$ & $253(24.7 \%)$ & .635 \\
\hline Performed in 2004 & $102(28.1 \%)$ & $247(24.1 \%)$ & .129 \\
\hline Performed in 2005 & $142(39.1 \%)$ & $283(27.6 \%)$ & $<.001$ \\
\hline
\end{tabular}

$A C E$, Angiotensin-converting enzyme; $C A D$, coronary artery disease; $C H F$, congestive heart failure; $C O P D$, chronic obstructive pulmonary disease; $C V A$, cerebrovascular accident; $I A B P$, intraaortic balloon pump; NYHA, New York Heart Association.

in the generalized estimating equation models as covariates. We excluded operative deaths without stroke or PMI from the models for stroke and PMI, respectively. Statistical analysis was performed with SAS version 9.1 (SAS Institute Inc, Cary, NC).

\section{RESULTS \\ Preoperative Characteristics}

Patient characteristics are shown in Table 2. Patients in the statin group were older and had higher body mass index, higher smoking frequency, hypertension, diabetes, chronic obstructive pulmonary disease, and cerebrovascular accident. They had more diagnoses of aortic valve disease and fewer diagnoses of mitral valve disease. In addition, there was a higher frequency of preoperative use of beta-blockers, angiotensin-converting enzyme inhibitors, and aspirin, and more procedures were performed in 2005. 


\section{Operative Outcomes}

The crude operative mortality was $0.8 \%$ (3/363) and $2.3 \%(24 / 1,026)$, the crude incidence of stroke was $1.7 \%$ $(6 / 360)$ and $2.9 \%(29 / 1007)$, and the crude incidence of PMI was $2.2 \%(8 / 362)$ and $2.4 \%(24 / 1005)$ in the statin and non-statin groups, respectively.

Generalized estimating equations showed that preoperative statin use was associated with lower mortality (odds ratio: $0.25,95 \%$ confidential interval: $0.12-0.54, P=.0004$ ) and not significantly associated with an incidence of stroke (odds ratio: $0.48,95 \%$ confidential interval: $0.19-1.22$, $P=.1215$ ) or PMI (odds ratio: $0.91,95 \%$ confidential interval: $0.43-1.91, P=.8025)$ in this cohort.

\section{Comment}

The mortality and morbidity of patients undergoing cardiac surgery is declining with improved surgical techniques, technologies, and perioperative care. Previous studies indicate that preoperative administration of statins could be a strategy for improving CABG outcomes. ${ }^{10-12}$ However, whether preoperative statins improve the operative outcomes of patients undergoing cardiac surgery with no history of CAD is unknown.

Clark and colleagues ${ }^{16}$ performed an 8 -year retrospective cohort study and reported beneficial effects of preoperative statins on the mortality and morbidity in patients undergoing various assortments of cardiac surgical procedures $(80 \%$ of patients underwent $\mathrm{CABG}$ ). In the subgroup of patients undergoing valve replacement or repair only, patients receiving statins had a lower mortality than patients not receiving statins $(1.96 \%$ vs $7.5 \%)$. However, this study's subgroup analysis was not statistically powerful and the history of CAD was not sufficiently clarified to be capable of demonstrating a benefit in patients without CAD.

We have shown a significant association of preoperative statin use with lower operative mortality in patients without CAD who underwent cardiac valve surgery. Preoperative statin use was not significantly associated with a lower incidence of new stroke; however, the results of a multivariable analysis (odds ratio, 0.48 ; $95 \%$ confidential interval, 0.19 1.22) imply that further investigation with a larger sample size or different study design would be worthwhile. There was no association between preoperative statin use and the incidence of PMI in this cohort.

The pleiotropic effects of statins may be an important biological mechanism underlying the beneficial effect of preoperative statins, although this is not causally proven and would warrant further investigation. The pleiotropic effects include improving endothelial function, enhancing the stability of atherosclerotic plaques, decreasing oxidative stress and inflammation, and inhibiting the thrombogenic response. ${ }^{1}$ These effects may attenuate the proinflammatory effects of surgical invasiveness and $\mathrm{CPB}$. The exposure to $\mathrm{CPB}$ causes a systemic inflammatory reaction culminating in organ injury and impaired function. Strategies to minimize CPB-related systemic inflammatory reactions include improvement of artificial surface biocompatibility, temporary inhibition of blood cell activation, and blockage of the bioactivities and effects of inflammatory mediators. ${ }^{17}$ Statins are known to suppress inflammatory mediators. Recent studies have shown that preoperative statin use suppresses serum levels of inflammatory markers after CABG. ${ }^{18-20}$

Stroke is a major complication after cardiac surgery. Mechanisms of perioperative stroke include cerebral hypoperfusion and thromboembolism. Statins have been shown to reduce brain injury in animal models of cerebral ischemia by up-regulating endothelial nitric oxide synthase. ${ }^{21} \mathrm{~A}$ previous study showed that preoperative statin use improves endothelial function after $\mathrm{CABG} .{ }^{22}$ Anti-inflammatory and antioxidant effects of statins can also be neuroprotective in cerebral ischemia. ${ }^{21}$ In addition, there is strong evidence that statins accelerate the breakdown of proinflammatory extracellular nucleotides and promote the formation of anti-inflammatory adenosine, a well-known and potent neuroprotectant. ${ }^{23}$ Clinically, preoperative statins have been shown to reduce perioperative stroke in patients undergoing CABG. ${ }^{24}$ Although a neuroprotective effect of statins in non-CABG cardiac surgery has not been reported, it seems biologically reasonable.

The majority of the patients in our cohort who were taking statins preoperatively were doing so because of their history of hypercholesterolemia. Decreasing serum cholesterol levels because of preoperative use of statins could be a reason for the beneficial effect that was observed. We did not include preoperative serum cholesterol levels in the analysis because they were not measured in all patients.

Clinically, this study implies that a preemptive use of preoperative statins may improve operative outcomes in patients undergoing cardiac valve surgery who do not have a history of CAD. Our cohort includes concomitant aortic, congenital, and other cardiac procedures. Combined with previous findings in CABG and patients with $\mathrm{CAD}$, the beneficial effects of preoperative statins may be generalizable to most patients undergoing cardiac surgery.

A limitation of this study is its observational nature. However, we minimized confounding effects by using the multivariable and propensity analyses. There are potential confounders we did not include in the propensity score (eg, pulmonary artery pressure, cardiac index, and use of aprotinin). Furthermore, this is a retrospective, single-center study. The clinical impact of these results may warrant a future prospective multicenter randomized controlled trial. Our study did not identify the optimal dose of preoperative statins because our patients were receiving various classes and doses of statins. Previous randomized controlled studies have shown that the administration of atorvastatin $20 \mathrm{mg} /$ $\mathrm{d}$ for 3 weeks before surgery has beneficial effects in patients 
undergoing CABG. ${ }^{19,22}$ Another randomized controlled study has shown that administration of atorvastatin $40 \mathrm{mg} /$ $\mathrm{d}$ for 7 days before surgery reduced the incidence of postoperative atrial fibrillation. ${ }^{25}$ Other studies have shown that 1 to 5 days of use of statins may improve endothelial function, ${ }^{26}$ reduce inflammation, ${ }^{27}$ and improve outcome. Further investigations are necessary to determine the optimal dose of preoperative statins.

\section{CONCLUSIONS}

Preoperative use of statins is associated with lower operative mortality in patients without CAD who underwent cardiac valve surgery. Preoperative statins may be useful for improving operative outcomes in patients without CAD who undergo cardiac surgery.

We thank M. Letti Byrne for assistance with data collection and management. We extend our gratitude to Dr E. John Orav for kind and generous efforts and assistance with this article's preparation.

\section{References}

1. Liao JK. Effects of statins on 3-hydroxy-3-methylglutaryl coenzyme A reductase inhibition beyond low-density lipoprotein cholesterol. Am J Cardiol. 2005; 96(suppl):24F-33.

2. Baigent C, Keech A, Kearney PM, et al. Efficacy and safety of cholesterol-lowering treatment: prospective meta-analysis of data from 90,056 participants in 14 randomised trials of statins. Lancet. 2005;366:1267-78.

3. Scandinavian Simvastatin Survival Study Group. Randomised trial of cholesterol lowering in 4444 patients with coronary heart disease: the Scandinavian Simvastatin Survival Study (4S). Lancet. 1994;344:1383-9.

4. Sacks FM, Pfeffer MA, Moye LA, et al. The effect of pravastatin on coronary events after myocardial infarction in patients with average cholesterol levels. Cholesterol and Recurrent Events Trial investigators. N Engl J Med. 1996;335:1001-9.

5. The Long-Term Intervention with Pravastatin in Ischaemic Disease (LIPID) Study Group. Prevention of cardiovascular events and death with pravastatin in patients with coronary heart disease and a broad range of initial cholesterol levels. The Long-Term Intervention with Pravastatin in Ischaemic Disease (LIPID) Study Group. N Engl J Med. 1998;339:1349-57.

6. Heart Protection Study Collaborative Group. MRC/BHF Heart Protection Study of cholesterol lowering with simvastatin in 20,536 high-risk individuals: a randomised placebo-controlled trial. Lancet. 2002;360:7-22.

7. Schwartz GG, Olsson AG, Ezekowitz MD, et al. Myocardial Ischemia Reduction with Aggressive Cholesterol Lowering (MIRACL) Study Investigators. Effects of atorvastatin on early recurrent ischemic events in acute coronary syndromes: the MIRACL study: a randomized controlled trial. JAMA. 2001; 285:1711-8.

8. Liem AH, van Boven AJ, Veeger NJ, et al. Fluvastatin On Risk Diminishment after Acute myocardial infarction study group. Effect of fluvastatin on ischaemia following acute myocardial infarction: a randomized trial. Eur Heart J. 2002; 23:1893-6.

9. Thompson PL, Meredith I, Amerena J, Campbell TJ, Sloman JG, Harris PJ. Pravastatin in Acute Coronary Treatment (PACT) Investigators. Effect of pravastatin compared with placebo initiated within 24 hours of onset of acute myocardial infarction or unstable angina: the Pravastatin in Acute Coronary Treatment (PACT) trial. Am Heart J. 2004; 148:e2.

10. Dotani MI, Elnicki DM, Jain AC, Gibson CM. Effect of preoperative statin therapy and cardiac outcomes after coronary artery bypass grafting. Am J Cardiol. 2000;86:1128-30.

11. Pan W, Pintar T, Anton J, Lee VV, Vaughn WK, Collard CD. Statins are associated with a reduced incidence of perioperative mortality after coronary artery bypass graft surgery. Circulation. 2004;110(11 Suppl. 1):II45-9.

12. Collard CD, Body SC, Shernan SK, Wang S, Mangano DT. Multicenter Study of Perioperative Ischemia (MCSPI) Research Group, Inc; Ischemia Research and Education Foundation (IREF) Investigators. Preoperative statin therapy is associated with reduced cardiac mortality after coronary artery bypass graft surgery. J Thorac Cardiovasc Surg. 2006;132:392-400.

13. Cepeda MS, Boston R, Farrar JT, Strom BL. Comparison of logistic regression versus propensity score when the number of events is low and there are multiple confounders. Am J Epidemiol. 2003;158:280-7.

14. D'Agostino RB. Propensity score methods for bias reduction in the comparison of a treatment to a non-randomized control group. Stat Med. 1998;17:2265-81.

15. Hanley JA, Negassa A, Edwardes MD, Forrester JE. Statistical analysis of correlated data using generalized estimating equations: an orientation. Am J Epidemiol. 2003; 157:364-75.

16. Clark LL, Ikonomidis JS, Crawford FA Jr, et al. Preoperative statin treatment is associated with reduced postoperative mortality and morbidity in patients undergoing cardiac surgery: an 8-year retrospective cohort study. J Thorac Cardiovasc Surg. 2006;131:679-85.

17. Li S, Price R, Phiroz D, Swan K, Crane TA. Systemic inflammatory response during cardiopulmonary bypass and strategies. J Extra Corpor Technol. 2005;37: 180-8.

18. Chello M, Patti G, Candura D, et al. Effects of atorvastatin on systemic inflammatory response after coronary bypass surgery. Crit Care Med. 2006;34:660-7.

19. Chello M, Carassiti M, Agro F, et al. Simvastatin blunts the increase of circulating adhesion molecules after coronary artery bypass surgery with cardiopulmonary bypass. J Cardiothorac Vasc Anesth. 2004;605-9.

20. Liakopoulos OJ, Dorge H, Schmitto JD, Nagorsnik U, Grabedunkel J, Schoendube FA. Effects of preoperative statin therapy on cytokines after cardiac surgery. Thorac Cardiovasc Surg. 2006;54:250-4.

21. Vaughan CJ, Delanty N. Neuroprotective properties of statins in cerebral ischemia and stroke. Stroke. 1999;30:1969-73.

22. Chello M, Goffredo C, Patti G, et al. Effects of atorvastatin on arterial endothelial function in coronary bypass surgery. Eur J Cardiothorac Surg. 2005;28: 805-10.

23. Osman L, Amrani M, Isley C, Yacoub MH, Smolenski RT. Stimulatory effects of atorvastatin on extracellular nucleotide degradation in human endothelial cells. Nucleosides Nucleotides Nucleic Acids. 2006;25:1125-8.

24. Aboyans V, Labrousse L, Lacroix P, et al. Predictive factors of stroke in patients undergoing coronary artery bypass grafting: statins are protective. Eur J Cardiothorac Surg. 2006;30:300-4.

25. Patti G, Chello M, Candura D, et al. Randomized trial of atorvastatin for reduction of postoperative atrial fibrillation in patients undergoing cardiac surgery: results of the ARMYDA-3 (Atorvastatin for Reduction of MYocardial Dysrhythmia After cardiac surgery) study. Circulation. 2006;114:1455-61.

26. Wassmann S, Faul A, Hennen B, Scheller B, Bohm M, Nickenig G. Rapid effect of 3-hydroxy-3-methylglutaryl coenzyme A reductase inhibition on coronary endothelial function. Circ Res. 2003;93:e98-103.

27. Correia LC, Sposito AC, Lima JC, et al. Anti-inflammatory effect of atorvastatin $(80 \mathrm{mg})$ in unstable angina pectoris and non-Q-wave acute myocardial infarction. Am J Cardiol. 2003;92:298-301. 